\title{
Clinicopathological Evaluation of the Potential Anatomic Pathways of Systemic Metastasis from Primary Breast Cancer Suggests an Orderly Spread Through the Regional Lymph Nodes
}

\author{
S. David Nathanson, $\mathrm{MD}^{1}$, Shravan Leonard-Murali, $\mathrm{MD}^{1}$, Charlotte Burmeister, $\mathrm{MS}^{2}$, Laura Susick, $\mathrm{PhD}^{2}$, and \\ Patricia Baker, BHCS ${ }^{2}$ \\ ${ }^{1}$ Department of Surgery, Henry Ford Health System, Detroit, MI; ${ }^{2}$ Department of Public Health Sciences, Henry Ford \\ Health System, Detroit, MI
}

\begin{abstract}
Background. Two conflicting hypotheses as to how breast cancer (BC) accesses the systemic circulation dominated the 20th century and affected surgical treatment. We hypothesized that tumor lymphovascular invasion (LVI) at the primary tumor site favors lymphatic and not blood vessel, capillaries, and systemic metastases (Smets) are dependent upon regional lymph node (RLN) mets.

Methods. Data from BC patients undergoing RLN biopsy was professionally abstracted and maintained in a prospective, precisely managed, single-institution database. Associations of RLN, LVI, and Smets were estimated by univariate and multivariate backward logistic regression models and patient-affiliated demographic, clinicopathologic, treatment type, and molecular marker data.

Results. Of 3329 patients, followed 1-22 years (mean 7.8), 463 of 3329 (13.9\%) showed LVI, 742 of 3329 (22.3\%) had RLN mets, and 262 of 3329 (7.9\%) had Smets. Smets occurred in 52 of 252 (21\% with LVI+/RLN+); 116 of 2301 (5\% with LVI-/RLN-); 65 of 465 (14\% with LVI-/RLN+); and 17 of 207 (8\% with LVI+/RLN-), $p=0.021$ for association between LVI and Smets for
\end{abstract}

This study was selected for presentation at the American Society of Breast Surgeons, 2020; however, the meeting was canceled because of the COVID-19 pandemic.

(C) Society of Surgical Oncology 2020

First Received: 15 April 2020

Accepted: 30 June 2020;

Published Online: 27 July 2020

S. David Nathanson, MD

e-mail: dnathan1@hfhs.org
RLN + patients but not for RLN - patients $(p=0.051)$. Positive RLN, larger tumor size, and higher grade (all $p<0.001)$ were predictive of Smets by the multivariable model, whereas positive LVI was not.

Conclusions. LVI predicts RLN mets in BC. RLN is critical to Smets from BC, whereas LVI on its own is not. Smets occur significantly more commonly when both LVI and RLN mets occur together. LVI is, thus, likely to be primarily lymphatic invasion, and rarely, blood vessel invasion, supporting the Halsted paradigm. LVI and RLN together predict clinical outcome better than either alone.

The modern surgical approaches to breast cancer (BC) treatment are based on paradigm shifts in the 20th century, related primarily to the natural history of the disease. Halsted notably based the concept of the radical mastectomy on the belief that BC spreads to axillary lymph nodes and then to systemic sites through lymphovenous anastomoses in the neck. ${ }^{1}$ Fisher, based on mouse and human experiments and the discovery of peritumoral angiogenesis, changed that belief, hypothesizing that $\mathrm{BC}$ invades the systemic circulation early in the disease process. ${ }^{2-4}$ Randomized, clinical trials were designed to test, and ultimately prove, the concept that less locoregional surgery was at least as effective as radical breast surgery in controlling the local disease. ${ }^{2}$

Lymphovascular invasion (LVI), the identification of tumor cells in an endothelium-lined space in or around the primary $\mathrm{BC}$, has been assumed by many modern oncologists to be invasion into blood vessels. It seems likely that Fisher was influenced by the observation of angiogenesis when designing his animal and human studies, because tumor-induced lymphangiogenesis had not yet been 
identified in the 1970s. ${ }^{4}$ Lymphangiogenesis, the growth of new lymphatic capillaries in and around BCs, has since been demonstrated to be just as clearly a part of the progression of the disease as angiogenesis. ${ }^{5}$ Two large pathologic studies, using specific lymphatic endothelial markers, have clearly shown that LVI in BC is primarily into lymphatics and not blood vessel capillaries, supporting the Halsted and disputing the Fisher hypothesis. ${ }^{6,7}$ Furthermore, the progression of $\mathrm{BC}$ through the sentinel lymph node (SLN) before systemic spread was recently proven in mouse experiments. ${ }^{8,9}$

Debate regarding the Halsted and Fisher hypotheses focus on whether regional lymph node (RLN) metastases (mets) are necessary for systemic metastases (Smets) to occur. The question of whether LVI related to the primary tumor predicts mets to systemic sites in the absence of RLN mets is thus especially important. If the invasion associated with the primary tumor in the breast is mostly or exclusively into lymphatic capillaries, the pathway to the RLNs is anatomically logical, tumor cells would access the systemic circulation indirectly and Smets would occur after first passing through the nodes. In contrast, if tumor cells predominantly invade blood capillaries associated with the primary tumor in the breast, they would pass directly into the systemic circulation without going through the RLNs; under these circumstances Smets could occur without RLN mets and surgical removal of the RLNs might not be necessary.

Based on animal studies and prior clinical and experimental observations, we hypothesized that invading BC cells would most likely invade lymphatic capillaries, follow a pathway to the RLN, and invade through lymphovascular transfer in the first, or SLN into the systemic circulation. ${ }^{10-13} \mathrm{We}$ interrogated our own BC database, which was initiated in 1995 and has been abstracted continuously for the past 24 years. The database represents a prospective accumulation of more than $3000 \mathrm{BC}$ patients, all within one healthcare system, with accurate, complete and extensive follow-up, and almost no patients lost to follow-up, from which we had previously published results with fewer numbers. ${ }^{13}$

\section{METHODS}

Data were prospectively collected from patients with BC treated within our tertiary-care hospital system from 1995 to 2019. All the patients in this study were diagnosed and managed by a multidisciplinary team of BC physicians and ancillary staff in a vertically integrated academic health system. The data collection was approved, and annually reapproved, by the institutional review board.
Clinical variables included age at time of diagnosis, primary site surgery (mastectomy or lumpectomy), lymph node dissection method (complete axillary dissection [CALND] and/or SLN biopsy), treatment modalities used (chemotherapy, radiation, targeted, and hormonal therapy), survival status (alive with or without recurrence, or dead), cause of death if known, presence, site, and timing of distant mets if applicable. Neither clinically nor radiologically apparent RLN mets nor systemic metastatic disease (smets) were present at time of the original diagnosis.

While management of early stage BC has changed over the period of this study, this study does not depend on types of surgical or adjuvant treatment because it looks at two events primarily: the status of the RLNs at initial treatment, and the presence or absence of lymphovascular invasion (LVI), and relates this information to the later development of systemic metastasis. Most current patients are offered SLN biopsy alone; CALND is used much less commonly in the past decade, but the first few hundred patients in the middle 1990s had both SLNB and CALND.

Specialist pathologic evaluation by a team of breast pathologists of all primary tumors and lymph nodes was done using standard pathologic protocols and reported electronically using a checklist as previously described. ${ }^{13}$ Briefly, the checklist included: anatomic part(s) of the breast involved, histopathologic type, histologic grade (Nottingham), maximum primary tumor size (centimeters), lympho-vascular invasion (present or absent), breast tumor markers (estrogen, progesterone, and HER-2-neu receptors; positive or negative, allowing subgroup analysis of triple negative disease), lymph nodes (number with metastasis/ number examined), largest node metastasis size (centimeters), extracapsular extension, and distant metastasis (yes or no) and pTNM stage.

Lymphovascular invasion (LVI) was scored unequivocally positive in tumor sections stained with hematoxylin and eosin in only those patients with unmistakable clusters of tumor cells in the lumen of an endothelial-lined vascular space within or close to the edge of the $\mathrm{BC}$ as a standard pathological evaluation, based upon American Society of Clinical and Anatomic Pathology (ASCAP) guidelines without special immunostain markers to identify endothelial cells.

Lymph nodes, found by either SLN biopsy alone or by CALND, were fixed in $10 \%$ buffered formalin and embedded in paraffin, serially sectioned along the short axis into 2-mm slices, and 4-6 sections, 5-micron thick, cut at various depths, and standard hematoxylin and eosin staining on glass slides performed. Cytokeratin immunohistochemistry (IHC) was used only to confirm nanometastases in the node, particularly with primary lobular carcinomas. An axillary node was classified as positive (containing metastatic tumor cells) according to standard 
pathologic criteria and current American Joint Commission on Cancer guidelines. The SLN is by definition the first RLN to harbor metastasis when this occurs.

If a patient had surgery on both sides on the same date, the side with the positive RLN or with collected RLN data (if no positive RLN was found) was used. Patients with invasive ductal or lobular carcinoma were included. A total of 253 patients with only DCIS who had mastectomies and RLN biopsies were excluded. Twenty-two patients with missing RLN data also were excluded. Chest wall recurrence alone was not considered Smets.

Frequently audited and updated data of all patients undergoing axillary node surgery for invasive BC, a remarkably stable, ethnically heterogeneous population in a unique group practice, was collected by trained abstractors for the entire collection period. This included identifying dates and sites of systemic metastasis.

Demographic and clinicopathologic variables were analyzed for frequency. Univariate and multivariable logistic regression models were constructed for RLN mets and Smets. For the multivariable models, individual predictors from the univariate models with $p<0.10$ were candidates for multivariable modeling. A backwards model selection method was used to determine the final multivariable model. Variables in the final model were significant at $p<0.05$. Odds ratios (OR) versus reference groups with $95 \%$ CI were calculated for all logistic regression models (by $\mathrm{CB}$ ).

Associations between LVI, RLN mets, and Smets were tested using $\chi^{2}$ test of independence. Tables were constructed for RLN negative, RLN positive, LVI negative, and LVI positive patients. Kaplan-Meier curves were constructed estimating time to Smets for patients in different combinations of LVI and RLN status.

\section{RESULTS}

There were 3329 patients who met the inclusion criteria. Ninety patients had two surgeries or procedures on both sides. Follow-up ranged from $<1$ year to 22 years, with a median follow-up of 7.8 years. Demographic information is shown in Table 1 . The majority $(82.5 \%)$ of patients were older than aged 50 years, had tumors mostly less than $5 \mathrm{~cm}$ in size $(<1 \mathrm{~cm}=26.5 \%, 1-2 \mathrm{~cm}=42.1 \%, 2-5 \mathrm{~cm}=$ $27.6 \%,>5 \mathrm{~cm}=2.8 \%$, and unmeasurable $=1.1 \%$, and of variable grade (grade $1=24.6 \%$, grade $2=46.3 \%$, grade $3=28.2 \%$, and not graded $=0.9 \%$ ). Most tumors were hormone receptor-positive $($ ER-positive $=80.5 \%$ and PR-positive $=74.0 \%$ ) and HER2/neu-negative $(74.0 \%)$. Mastectomy was performed in $26.3 \%$ of patients $(73.7 \%$ had lumpectomies); $27.2 \%$ underwent CALND, $13.9 \%$ were LVI positive, $22.3 \%$ were RLN positive, and $7.9 \%$ were Smets positive.

Patient subgroups, with proportions based on LVI, RLN, and Smets status, is shown in Fig. 1.

The univariate logistic regression model and multivariable backwards logistic regression model for associations of variables with RLN positive status are shown in Table 2. Based on the univariate model, the multivariable model for associations of variables with RLN positive status initially included HER2/neu status, ER status, PR status (including

TABLE 1 Demographic and clinicopathologic data of included patients

\begin{tabular}{|c|c|}
\hline Variable & Total $(\%)$ \\
\hline Total cases & $3329(100.0 \%)$ \\
\hline \multicolumn{2}{|l|}{ Age } \\
\hline$<50$ & $580(17.5 \%)$ \\
\hline$\geq 50$ & $2740(82.5 \%)$ \\
\hline \multicolumn{2}{|l|}{ Tumor size $(\mathrm{cm})$} \\
\hline$<1$ & $881(26.5 \%)$ \\
\hline $1-2$ & $1403(42.1 \%)$ \\
\hline $2-5$ & $917(27.6 \%)$ \\
\hline$>5$ & $93(2.8 \%)$ \\
\hline Unmeasurable & $35(1.1 \%)$ \\
\hline \multicolumn{2}{|l|}{ Tumor grade } \\
\hline 1 & $818(24.6 \%)$ \\
\hline 2 & $1537(46.3 \%)$ \\
\hline 3 & $936(28.2 \%)$ \\
\hline Not graded & $30(0.9 \%)$ \\
\hline \multicolumn{2}{|l|}{ Pathology } \\
\hline IDCA & $2913(87.5 \%)$ \\
\hline ILCA & $426(12.8 \%)$ \\
\hline \multicolumn{2}{|l|}{ Hormone receptor status } \\
\hline ER-positive & $2664(80.5 \%)$ \\
\hline PR-positive & $2456(74.0 \%)$ \\
\hline \multicolumn{2}{|l|}{ HER2/neu status } \\
\hline Positive & $579(17.5 \%)$ \\
\hline Negative & $2452(74.0 \%)$ \\
\hline Not performed & $284(8.6 \%)$ \\
\hline \multicolumn{2}{|l|}{ Surgery type } \\
\hline Mastectomy & $871(26.3 \%)$ \\
\hline Full axillary dissection & $890(27.2 \%)$ \\
\hline Chemotherapy & $1494(45.4 \%)$ \\
\hline LVI & $463(13.9 \%)$ \\
\hline RLN positive & $742(22.3 \%)$ \\
\hline Systemic metastasis & $262(7.9 \%)$ \\
\hline
\end{tabular}

$E R$ estrogen receptor; IDCA invasive ductal carcinoma; ILCA invasive lobular carcinoma; $P R$ progesterone receptor; $R L N$ regional lymph node 
triple-negative), tumor size, grade, LVI, age, and Smets status. HER2/neu, ER, and PR were excluded in the backwards selection model. No significant interactions were found. Predictors in this model of RLN-positive status were age $<50$ years (odds ratio [OR] 1.61 vs. reference group of age $\geq 50,95 \%$ confidence interval [CI] 1.27-2.05; $p<0.001$ ); tumor size $1-2 \mathrm{~cm}$ (OR 2.61 vs. reference tumor size $<1 \mathrm{~cm}, 95 \%$ CI 1.92-3.54; $p<0.001)$ and $2-5 \mathrm{~cm}$ (OR 4.16 vs. reference tumor size $<1 \mathrm{~cm}, 95 \%$ CI 3.03-5.70; $p<0.001)$; tumor grade of 2 (OR 1.66 vs. reference of tumor grade of 1, 95\% CI $1.27-2.16$; $p<0.001$ ); presence of LVI (OR 4.73 vs. reference group of no LVI, 95\% CI 3.73-5.99; $p<0.001)$; and presence of Smets (OR 2.34 vs. reference group of no Smets, 95\% CI 1.71-3.20; $p<0.001)$.

The univariate logistic regression model and multivariable backwards logistic regression model for associations of variables with Smets positive status are shown in Table 3. Based on the univariate model, the multivariable model for associations of variables with Smets-positive status initially included RLN status, HER2/neu status, ER status, PR status, tumor size, grade, LVI, and age. HER2/ neu status, ER status, PR status, LVI, and age were excluded in the backwards selection model. No significant interactions were found. Predictors in this model of Smets positive status were tumor size $1-2 \mathrm{~cm}$ (OR 1.69 vs. reference tumor size $<1 \mathrm{~cm}, 95 \%$ CI $1.05-2.74 ; p=0.033$ ) and $2-5 \mathrm{~cm}$ (OR 2.95 vs. reference tumor size $<1 \mathrm{~cm}$,
95\% CI 1.82-4.78; $p<0.001$ ); tumor grade of 3 (OR 2.33 vs. reference of tumor grade of 1 , 95\% CI 1.48-3.65; $p<0.001$ ); and positive RLN (OR 2.49 vs. reference group of negative RLN, 95\% CI 1.85-3.34; $p<0.001)$.

Table 4 shows results of $\chi^{2}$ test of independence to determine associations between LVI and Smets among RLN-negative and RLN-positive patients. There was no significant association between LVI and Smets for RLNnegative patients $(p=0.051)$. There was a significant association between LVI and Smets for RLN-positive patients $(p=0.021)$.

Table 5 shows results of $\chi^{2}$ test of independence to determine associations between LVI and RLN mets among Smets-negative and Smets-positive patients. There was a significant association between LVI and RLN metastasis for Smets-negative patients $(p<0.001)$. There also was a significant association between LVI and RLN metastasis for Smets-positive patients $(p<0.001)$.

Figure 2 shows Kaplan-Meier curves of time to Smets for the different LVI/RLN-positive and negative subgroups. No group reached median time to Smets. At the end point of 22 years, LVI-positive/RLN-positive patients had the highest incidence of Smets, while LVI-negative/ RLN-negative patients had the lowest incidence of Smets. LVI-negative/RLN-positive patients had a higher incidence of Smets than LVI-positive/RLN-negative patients.

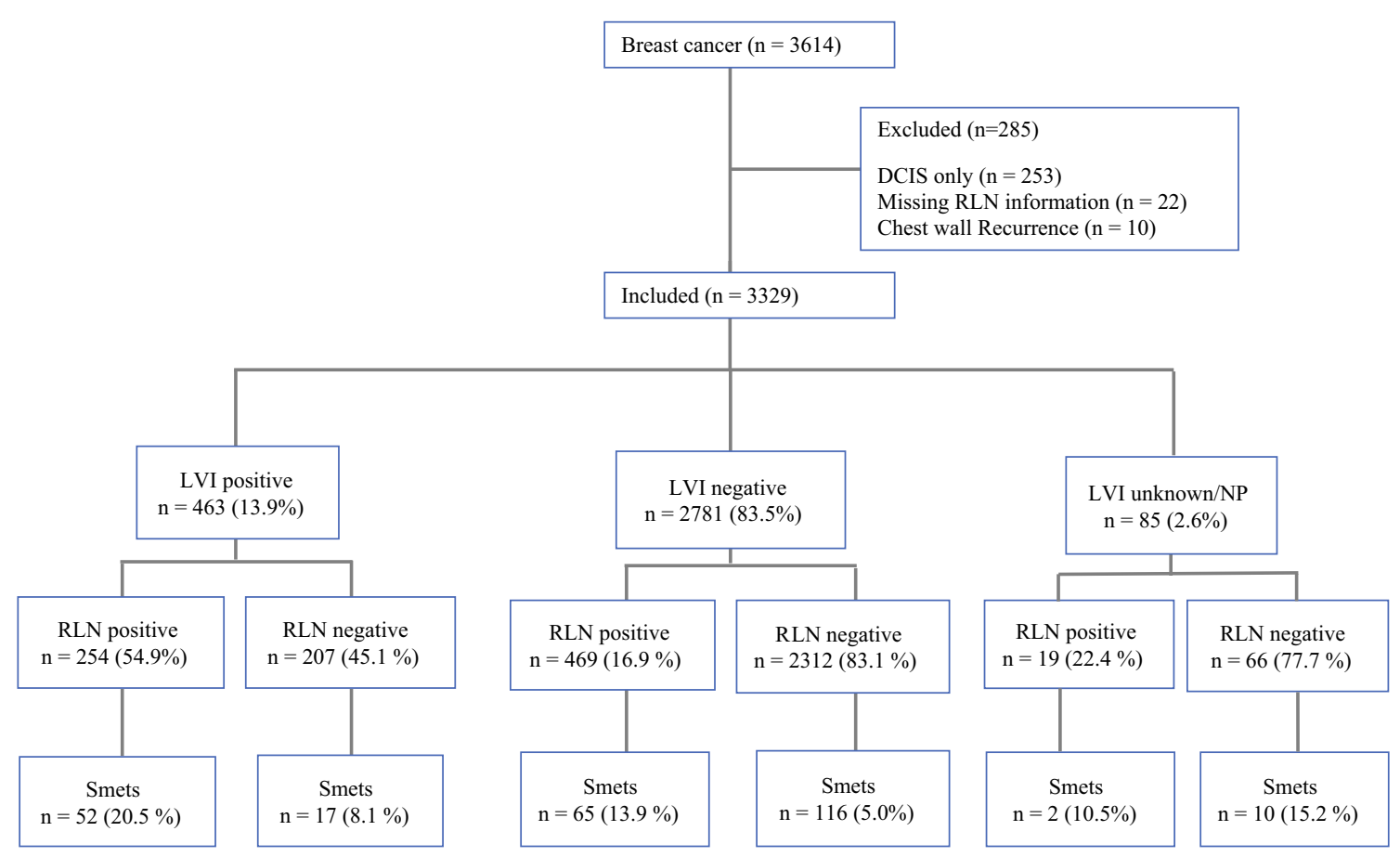

FIG. 1 Cohort selection, with proportions of LVI, RLN, and Smets statuses. DCIS ductal carcinoma in situ; LVI lymphovascular invasion; RLN regional lymph node; Smets systemic metastases 
TABLE 2 Univariate logistic regression model and multivariable backwards logistic regression model for associations of variables with RLN positivity

\begin{tabular}{|c|c|c|c|c|c|c|}
\hline \multirow[t]{2}{*}{ Variable } & \multirow[t]{2}{*}{ RLN metastasis $(n=742)$} & \multirow[t]{2}{*}{ No RLN metastasis $(n=2587)$} & \multicolumn{2}{|l|}{ Univariate analysis } & \multicolumn{2}{|l|}{ Multivariable analysis } \\
\hline & & & Odds ratio $(95 \% \mathrm{CI})$ & $p$ value & Odds ratio $(95 \% \mathrm{CI})$ & $p$ value \\
\hline \multicolumn{7}{|l|}{ Age (year) } \\
\hline$\geq 50$ & $569(77 \%)$ & $2171(84 \%)$ & Reference & & Reference group & \\
\hline$<50$ & $173(23 \%)$ & $407(16 \%)$ & $1.62(1.33,1.98)$ & $<0.001$ & $1.61(1.27,2.05)$ & $<0.001$ \\
\hline \multicolumn{7}{|c|}{ Tumor size $(\mathrm{cm})$} \\
\hline$<1$ & $71(10 \%)$ & $810(32 \%)$ & Reference & & Reference group & \\
\hline $1-2$ & $301(41 \%)$ & $1102(43 \%)$ & $3.12(2.37,4.10)$ & $<0.001$ & $2.61(1.92,3.54)$ & $<0.001$ \\
\hline $2-5$ & $363(49 \%)$ & $647(25 \%)$ & $6.40(4.86,8.42)$ & $<0.001$ & $4.16(3.03,5.70)$ & $<0.001$ \\
\hline \multicolumn{7}{|l|}{ Grade } \\
\hline 1 & $105(14 \%)$ & $713(28 \%)$ & Reference & & Reference group & \\
\hline 2 & $396(54 \%)$ & $1141(45 \%)$ & $2.36(1.86,2.98)$ & $<0.001$ & $1.66(1.27,2.16)$ & $<0.001$ \\
\hline 3 & $231(32 \%)$ & $705(28 \%)$ & $2.23(1.73,2.87)$ & $<0.001$ & $0.99(0.73,1.34)$ & 0.928 \\
\hline \multicolumn{7}{|l|}{ LVI } \\
\hline Negative & $469(65 \%)$ & $2312(92 \%)$ & Reference & & Reference group & \\
\hline Positive & $254(35 \%)$ & $209(8 \%)$ & $5.99(4.87,7.38)$ & $<0.001$ & $4.73(3.73,5.99)$ & $<0.001$ \\
\hline \multicolumn{7}{|c|}{ Systemic metastasis } \\
\hline No & $616(84 \%)$ & $2431(94 \%)$ & Reference & & Reference group & \\
\hline Yes & $119(16 \%)$ & $143(6 \%)$ & $3.28(2.54,4.25)$ & $<0.001$ & $2.34(1.71,3.20)$ & $<0.001$ \\
\hline \multicolumn{7}{|l|}{ ER } \\
\hline Negative & $124(17 \%)$ & $515(20 \%)$ & Reference & & & \\
\hline Positive & $613(83 \%)$ & $2051(80 \%)$ & $1.24(1.00,1.54)$ & 0.050 & & \\
\hline \multicolumn{7}{|l|}{ PR } \\
\hline Negative & $174(24 \%)$ & $683(27 \%)$ & Reference & & & \\
\hline Positive & $565(76 \%)$ & $1891(73 \%)$ & $1.17(0.97,1.42)$ & 0.102 & & \\
\hline \multicolumn{7}{|l|}{ HER2/neu } \\
\hline Negative & $512(77 \%)$ & $1940(82 \%)$ & Reference & & & \\
\hline Positive & $152(23 \%)$ & $427(18 \%)$ & $1.35(1.09,1.66)$ & 0.005 & & \\
\hline
\end{tabular}

$E R$ estrogen receptor; $L V I$ lymphovascular invasion; $P R$ progesterone receptor; $R L N$ regional lymph node

\section{DISCUSSION}

LVI was significantly associated with RLN mets in this study of 3329 clinically node-negative and initially smetsnegative patients with invasive BC offered standard surgical management in the form of lumpectomy/partial mastectomy or total mastectomy plus SLN biopsy and/or CALND, and adjuvant therapy, and followed for $<1-22$ years (median 7.8 years). Analysis also showed that Smets in the presence of LVI usually required mets to the RLNs for the association to be true, supporting the likelihood that the invaded vessels were lymphatics carrying tumor cells to RLNs, rather than blood vessels.

Of 463 patients with positive RLNs only 254 (55\%) were LVI-positive. An unknown proportion of the 209 patients LVI-positive/RLN-negative may have had pathologically unrecognized RLN mets; routine histologic examination of axillary lymph nodes in patients with $\mathrm{BC}$ significantly underestimates RLN mets. ${ }^{13,14}$ This histologic sampling error may occur in up to $30 \%$ of RLN negative cases. A similar sampling error may account for the 469 LVI-negative patients with RLN mets; if every adjacent micron of the tumor were evaluated, LVI would likely be found in a much higher number. In patients where Smets occurred in the absence of RLN mets, it is possible that many of those called RLN-negative were likely to have had missed RLN mets. ${ }^{13}$

Proof of independent RLN and hematogenous metastases has been debated using clinical, pathological, and genomic approaches. ${ }^{15}$ Preclinical and clinical studies suggest that capillary invasion is the preferred initial step whereby tumor cells interact with intratumoral or peritumoral vessels, metastasize to the RLNs and gain access to the systemic circulation following invasion of blood 
TABLE 3 Univariate logistic regression model and multivariable backwards logistic regression model for associations of variables with systemic metastasis positivity

\begin{tabular}{|c|c|c|c|c|c|c|}
\hline \multirow[t]{2}{*}{ Variable } & \multirow[t]{2}{*}{ RLN metastasis $(n=742)$} & \multirow[t]{2}{*}{ No RLN metastasis $(n=2587)$} & \multicolumn{2}{|l|}{ Univariate analysis } & \multicolumn{2}{|l|}{ Multivariable analysis } \\
\hline & & & Odds ratio $(95 \% \mathrm{CI})$ & $p$ value & Odds ratio $(95 \% \mathrm{CI})$ & $p$ value \\
\hline \multicolumn{7}{|l|}{ Age (year) } \\
\hline$\geq 50$ & $208(79 \%)$ & $2514(83 \%)$ & Reference & & & \\
\hline$<50$ & $54(21 \%)$ & $524(17 \%)$ & $1.25(0.91,1.71)$ & 0.170 & & \\
\hline \multicolumn{7}{|c|}{ Tumor size $(\mathrm{cm})$} \\
\hline$<1$ & $32(13 \%)$ & $843(28 \%)$ & Reference Group & & Reference group & \\
\hline $1-2$ & $91(36 \%)$ & $1303(43 \%)$ & $1.84(1.22,2.78)$ & 0.004 & $1.69(1.05,2.74)$ & 0.033 \\
\hline $2-5$ & $133(52 \%)$ & $873(29 \%)$ & $4.01(2.70,5.97)$ & $<0.001$ & $2.95(1.82,4.78)$ & $<0.001$ \\
\hline \multicolumn{7}{|l|}{ Grade } \\
\hline 1 & $33(13 \%)$ & $778(26 \%)$ & Reference Group & & Reference group & \\
\hline 2 & $113(44 \%)$ & $1416(47 \%)$ & $1.88(1.26,2.80)$ & 0.002 & $1.34(0.86,2.10)$ & 0.191 \\
\hline 3 & $113(44 \%)$ & $818(27 \%)$ & $3.26(2.18,4.86)$ & $<0.001$ & $2.33(1.48,3.65)$ & $<0.001$ \\
\hline \multicolumn{7}{|l|}{ LVI } \\
\hline Negative & $181(72 \%)$ & $2585(87 \%)$ & Reference group & & & \\
\hline Positive & $69(28 \%)$ & $390(13 \%)$ & $2.53(1.88,3.40)$ & $<0.001$ & & \\
\hline \multicolumn{7}{|l|}{ RLN } \\
\hline Negative & $143(55 \%)$ & $2431(80 \%)$ & Reference group & & Reference group & \\
\hline Positive & $119(45 \%)$ & $616(20 \%)$ & $3.28(2.54,4.25)$ & $<0.001$ & $2.49(1.85,3.34)$ & $<0.001$ \\
\hline \multicolumn{7}{|l|}{ ER } \\
\hline Positive & $193(74 \%)$ & $2452(81 \%)$ & Reference group & & & \\
\hline Negative & $67(26 \%)$ & $571(19 \%)$ & $1.49(1.11,2.00)$ & 0.008 & & \\
\hline \multicolumn{7}{|l|}{ PR } \\
\hline Positive & $177(68 \%)$ & $2263(75 \%)$ & Reference group & & & \\
\hline Negative & $82(32 \%)$ & $771(25 \%)$ & $1.36(1.03,1.79)$ & 0.028 & & \\
\hline \multicolumn{7}{|l|}{ HER2/neu } \\
\hline Negative & $182(79 \%)$ & $2253(81 \%)$ & Reference group & & & \\
\hline Positive & $48(21 \%)$ & $529(19 \%)$ & $1.12(0.81,1.57)$ & 0.492 & & \\
\hline
\end{tabular}

$E R$ estrogen receptor; $L V I$ lymphovascular invasion; $P R$ progesterone receptor; $R L N$ regional lymph node

TABLE $4 \chi^{2}$ test of independence of LVI and systemic metastasis among RLN-negative and RLN-positive patients

\begin{tabular}{|c|c|c|c|c|}
\hline \multirow{2}{*}{\multicolumn{2}{|c|}{ RLN-negative patients }} & \multicolumn{3}{|c|}{ Systemic metastasis } \\
\hline & & No & Yes & \\
\hline \multirow[t]{2}{*}{ LVI } & No & $2185(95 \%)$ & $116(5 \%)$ & $p$ value \\
\hline & Yes & $190(92 \%)$ & $17(8 \%)$ & 0.051 \\
\hline \multirow{2}{*}{\multicolumn{2}{|c|}{ RLN-positive patients }} & \multicolumn{3}{|c|}{ Systemic metastasis } \\
\hline & & No & Yes & \\
\hline \multirow[t]{2}{*}{ LVI } & No & $400(86 \%)$ & $65(14 \%)$ & $p$ value \\
\hline & Yes & $200(79 \%)$ & $52(21 \%)$ & 0.021 \\
\hline
\end{tabular}

There was no significant association between LVI and Smets for RLN-negative patients $(p=0.051)$. There was a significant association between LVI and Smets for RLN-positive patients $(p=0.021)$

$L V I$ lymphovascular invasion; $R L N$ regional lymph node
TABLE $5 \chi^{2}$ test of independence of LVI and RLN metastasis among systemic metastasis negative and systemic metastasis positive patients

\begin{tabular}{lclll}
\hline \multicolumn{2}{l}{ No systemic metastasis patients } & \multicolumn{4}{l}{ RLN metastasis } \\
& & No & Yes & \\
LVI & No & $2185(95 \%)$ & $116(5 \%)$ & $p$ value \\
& Yes & $400(86 \%)$ & $65(14 \%)$ & $<0.001$ \\
\multicolumn{4}{r}{ Systemic metastasis patients } & RLN metastasis \\
& & No & Yes & \\
LVI & No & $190(92 \%)$ & $17(8 \%)$ & $p$ value \\
& Yes & $200(79 \%)$ & $52(21 \%)$ & $<0.001$
\end{tabular}

There was a significant association between LVI and positive RLN for Smets negative patients $(p<0.001)$. There was also a significant association between LVI and positive RLN for Smets positive patients $(p<0.001)$

LVI lymphovascular invasion; $R L N$ regional lymph node 
FIG. 2 Kaplan-Meier curves of time to systemic metastasis for groups based on lymphovascular invasion and regional lymph node status. $L V I-$ lymphovascular invasion negative; $L V I+$ lymphovascular invasion positive; $R L N-$ regional lymph node negative; $R L N+$ regional lymph node positive

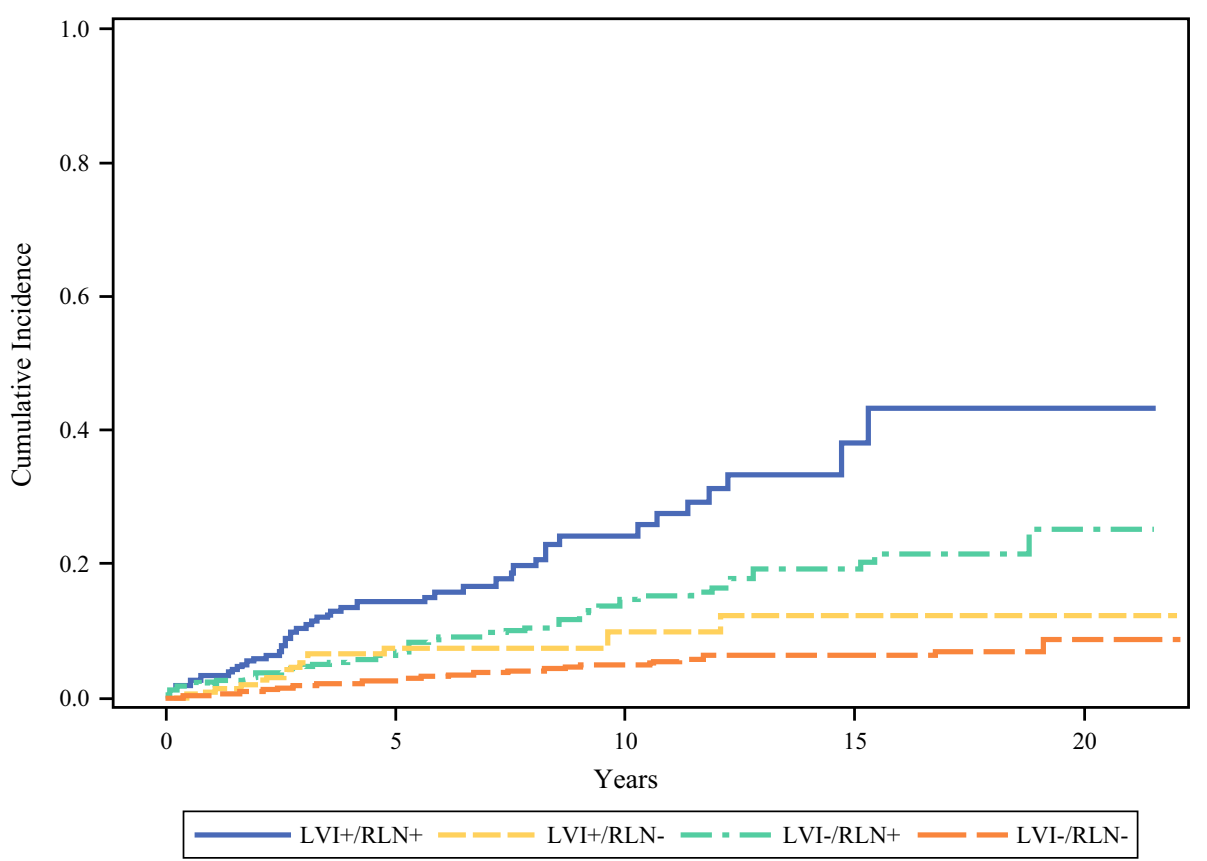

vessels. $^{13,16,17}$ When tumor-induced angiogenesis was discovered many observers extrapolated that LVI at the primary BC site indicated tumor cells invading into new blood vessels. $3,4,18,19$

Other sites of tumor cell invasion into the systemic circulation might be in the RLN or via the thoracic duct into the internal jugular vein, with a predominant pattern of orderly lymphatic and lymph node progression; ${ }^{8,9,20}$ Smets would follow in some patients. ${ }^{1}$

A direct association between lymphatic and hematogenous mets has been noted in many clinical studies. ${ }^{10,11,13,21}$ In the scientific literature diagrams depicting anatomic pathways of Smets show direct tumor invasion of blood vessels, with authors suggesting that angiogenesis provides a larger area of endothelium for tumor cells to invade and metastasize. $^{13,22}$ If tumor cells were to preferentially invade blood vessels, we would be hard pressed to explain how those tumors metastasize to the RLNs, because that probably requires direct lymphatic invasion.

The relative contributions to smets of direct intravasation of tumor cells into the bloodstream at the primary tumor site versus an indirect route via lymphatic capillaries as routes of egress from the primary site is still debated. $^{13,23}$ The orderly progression of common cancers through the lymphatic system to the bloodstream has certainly been described in animal tumor models, ${ }^{10,24}$ human melanoma, ${ }^{25}$ and in BC. ${ }^{1,13}$

Some animal tumor models show unequivocal preferential tumor cell invasion into peritumoral lymphatics by direct observation using videomicroscopy. ${ }^{8,9,26}$ Such undeniable real-time proof of lymphatic vessel invasive preference has yet to be demonstrated in human cancers, including BC. However, studies using highly specific lymphatic endothelial immunohistochemical stains allowed pathologists to observe direct $\mathrm{BC}$ tumor cell invasion into peritumoral lymphatics in close to $100 \%$ of cases when LVI was present. ${ }^{6,7,13}$ Other clinical and preclinical studies showed a correlation between RLN mets and lymphangiogenesis, suggesting a higher likelihood of lymphatic invasion with a larger surface area of lymphatic endothelium. $^{13,22,27-29}$

Pathophysiologic studies provide further evidence that tumor cells are more likely to invade peritumoral lymphatics. Interactions amongst endothelial cells, tumor, the tumor microenvironment, and microfluidic changes suggest a strong influence of interstitial fluid flow volumes and pressures and the invasion of lymphatics rather than blood vessels. ${ }^{30}$ Blood vessel capillaries exhibit interendothelial tight junctions, pericytes, and basement membranes, potential mechanical barriers to invading tumor cells not seen in new lymphatic vessels found in tumors. ${ }^{31,32}$ Abnormally functioning blood and lymphatic vessels in tumors result in significant fluid convection and edema near the tumor margin, ${ }^{28,33,34}$ compressing blood vessels, ${ }^{35}$ further decreasing the likelihood of tumor cell invasion. In contrast, lymphatic lumens may remain open despite the increased pressure, because of tethering filaments that bind lymphatic endothelial cells to surrounding stromal tissues, and mechanically enhance the intravasation of fluid and tumor cells, as noted previously. ${ }^{13}$ Tumor cells may initially be protected from mechanical destruction of the highshear blood circulation by first entering the low-shear 
lymphatic system and proliferating before being exposed to the systemic circulation. ${ }^{36}$ If more tumor cells enter the blood stream, more survive. ${ }^{10}$

There is strong evidence that tumor cells reaching RLNs can directly access the systemic circulation through lymphaticovenous invasion in the node. Direct lymphovenous connections in lymph nodes was first suggested by pathologists while looking at histologic sections. ${ }^{37}$ Radiologic studies in patients using air, bacteria, radioactive chromium, or radio-opaque contrast material, also showed lymphovenous connections in lymph nodes, as previously noted. ${ }^{13,38-40}$ Recent animal studies show tumor cells gaining access to the systemic circulation through the RLN. ${ }^{8,9}$

Neoadjuvant chemotherapy, more commonly used in the past decade than in the first decade of our database, could potentially decrease the incidence of LVI, RLN mets, and smets, but since we see no change in the statistics and conclusions of our current study compared with our study published 11 years ago, the conclusions were not affected by chemotherapy treatment. ${ }^{13}$

A potential weakness of our study, using prospectively accumulated data but retrospective in design, with level II evidence, might be enhanced by accumulating additional patients in a multi-institutional study.

Future studies using our data base and unique follow-up could include looking at genomic biomarkers from the stored paraffin blocks of this large cohort that might help to distinguish patients whose tumors may be more likely to invade blood vessels and not lymphatics. A related study could look at antibodies to specific lymphatic endothelial markers and enable oncologists to subclassify patients who might be able to avoid regional axillary node surgery if the markers suggest they are unlikely to develop RLN mets. ${ }^{6,7}$ This also could theoretically enable oncologists to select systemic therapies in those patients in whom the likelihood of smets is high based on these markers even when metastasis to RLNs has not occurred.

RLN mets treated by CALND or locoregional radiotherapy may produce long-term survival without Smets, suggesting a stage when tumor cells are confined to the breast and the RLNs and have not invaded the systemic blood stream or metastasized to visceral sites, as previously noted. $^{13,41}$ The findings of ACOSOG Z0011, which showed that there is no advantage to CALND versus SLN biopsy alone in early BC, would support the likelihood that invasion into blood vessels in the RLN probably requires both a threshold volume of tumor cells in the node and, perhaps, molecular mechanisms not yet identified. ${ }^{11}$

In summary, our data showed that LVI is a predictor of RLN mets, and when coupled with RLN mets, Smets. The likelihood of Smets was small and statistically insignificant when LVI was observed and where no RLN mets were found. RLN mets was an independent predictor of Smets, and the likelihood of Smets was significantly higher when both LVI and RLN mets occurred together. This information strongly supports the concept that primary BC gains access to the systemic circulation via invasion of lymphatic capillaries and an orderly spread through the RLNs. Direct invasion of the systemic circulation at the primary BC site may occur in a small number of patients, and it is likely that there are patients in whom both $\mathrm{BC}$ and $\mathrm{LC}$ have invasion. The ability to distinguish these different populations will be a challenge for future researchers.

ACKNOWLEDGMENT Supported by the Nathanson/Rands Chair in Breast Cancer Research and by The Team Angels Organization, Sterling Heights, Michigan

DISCLOSURE None.

\section{REFERENCES}

1. Halsted WS. The results of radical operations for the cure of carcinoma of the breast. Ann Surg. 1907;46(1):1-19.

2. Fisher B. Laboratory and clinical research in breast cancer-a personal adventure: the David A. Karnovsky memorial lecture. Cancer Res. 1980;40(11):3863-74.

3. Folkman J. Proceedings: tumor angiogenesis factor. Cancer Res. 1974;34(8):2109-13.

4. Folkman J. Role of angiogenesis in tumor growth and metastasis. Semin Oncol. 2002;29(6 Suppl 16):15-8.

5. Nathanson SD, Miller CG, Paxton JH. The role of lymphangiogenesis in regional lymph node metastasis: animal models. In: Leong SPL, editor. From local invasion to metastatic cancer: involvement of different sites through the lymphovascular system. New York: Humana Press; 2009. p. 211-226.

6. Mohammed RAA, Martin SG, Gill MS, Green AR, Paish EC, Ellis IO. Improved methods of detection of lymphovascular invasion demonstrate that it is the predominant method of vascular invasion in breast cancer and has important clinical consequences. Am J Surg Pathol. 2007;31(12):1825-33.

7. Schoppmann SF, Bayer G, Aumayr K, et al. Prognostic value of lymphangiogenesis and lymphovascular invasion in invasive breast cancer. Ann Surg. 2004;240(2):306-12.

8. Pereira ER, Kedrin D, Seano G, et al. Lymph node metastases can invade local blood vessels, exit the node, and colonize distant organs in mice. Science. 2018;359(6382):1403-7.

9. Brown M, Assen FP, Leithner A, et al. Lymph node blood vessels provide exit routes for metastatic tumor cell dissemination in mice. Science. 2018;359(6382):1408-11.

10. Nathanson SD. Preclinical models of regional lymph node tumor metastasis. In: Leong SPL, editor. Cancer metastasis and the lymphovascular system: basis for rational therapy. New York: Springer; 2007. p. 129-156.

11. Giuliano AE, Hunt KK, Ballman KV, et al. Axillary dissection versus no axillary dissection in women with invasive breast cancer and sentinel node metastasis: a randomized clinical trial. JAMA. 2011;305(6):569-75.

12. Fisher B, Jeong JH, Anderson S, Bryant J, Fisher ER, Wolmark $\mathrm{N}$. Twenty-five-year follow-up of a randomized trial comparing radical mastectomy, total mastectomy and total mastectomy followed by irradiation. N Engl J Med. 2002;347(8):567-75. 
13. Nathanson SD, Kwon D, Kapke A, Alford SH, Chitale D. The role of lymph node metastasis in the systemic dissemination of breast cancer. Ann Surg Oncol. 2009;16(12):3396-405.

14. Chagpar A, Middleton LP, Sahin AA, et al. Clinical outcome of patients with lymph node-negative breast carcinoma who have sentinel lymph node micrometastases detected by immunohistochemistry. Cancer. 2005;103(8):1581-6.

15. Yates LR, Gerstung M, Knappskog S, et al. Subclonal diversification of primary breast cancer revealed by multiregion sequencing. Nat Med. 2015;21(7):751-9.

16. Fisher E, Gregorio RM, Fisher B, Redmond C, Vellios F, Sommers SC. The pathology of invasive breast cancer. A syllabus derived from findings of the National Surgical Adjuvant Breast Project (protocol no. 4). Cancer. 1975;36(1):1-85.

17. Wong SY, Hynes RO. Lymphatic or hematogenous dissemination: how does a metastatic tumor cell decide? Cell Cycle. 2006;5(8):812-7.

18. Fidler IJ. Critical determinants of metastasis. Semin Cancer Biol. 2002;12(2):89-96.

19. Carmeliet P, Jain RK. Angiogenesis in cancer and other diseases. Nature. 2000;407(6801):249-57.

20. Virchow R, Chance F, Goodsir J, Osborn S. Cellular pathology as based upon physiological and pathological histology; twenty lectures delivered in the Pathological Institute of Berlin during the months of February, March and April, 1858. London: John Churchill; 1860.

21. Quiet CA, Ferguson DJ, Weichselbaum RR, Hellman S. Natural history of node-negative breast cancer: a study of 826 patients with long-term follow-up. J Clin Oncol. 1995;13(5):1144-51.

22. Liotta LA, Kleinerman J, Saidel GM. Quantitative relationships of intravascular tumor cells, tumor vessels, and pulmonary metastases following tumor implantation. Cancer Res. 1974;34(5):997-1004.

23. Nathanson SD. Insights into the mechanisms of lymph node metastasis. Cancer. 2003;98(2):413-23.

24. Yoshizawa M, Shingaki S, Nakajima T, Saku T. Histopathological study of lymphatic invasion in squamous carcinoma $(\mathrm{O}-1 \mathrm{~N})$ with high potential of lymph node metastasis. Clin Exp Metastasis. 1994;12(6):347-56.

25. Reintgen D, Cruse CW, Wells K, Berman C, Fenske N, Glass F, et al. The orderly progression of melanoma nodal metastases. Ann Surg. 1994;220:759-67.

26. Hoshida T, Isaka N, Hagendoorn J, et al. Imaging steps of lymphatic metastasis reveals that vascular endothelial growth factor$\mathrm{C}$ increases metastasis by increasing delivery of cancer cells to lymph nodes: therapeutic implications. Cancer Res. 2006;66(16):8065-75.

27. Nathanson SD, Zarbo RJ, Wachna DL, Spence CA, Andrzejewski TA, Abrams J. Microvessels that predict axillary lymph node metastases in patients with breast cancer. Arch Surg. 2000;135(5):586-93.

28. Hirakawa S, Kodama S, Kunstfield R, Kajiya K, Brown LF, Detmar M. VEGF-a induces tumor and sentinel lymph node lymphangiogenesis and promotes lymphatic metastasis. $J$ Exp Med. 2005;201(7):1089-99.

29. He Y, Rajantie I, Pajusola K, et al. Vascular endothelial growth factor receptor 3-mediated activation of lymphatic endothelium is crucial for tumor cell entry and spread via lymphatic vessels. Cancer Res. 2005;65(11):4739-46.

30. Greenlee JD, King MR. Engineered fluidic systems to understand lymphatic cancer metastasis. Biomicrofluidics. 2020;14(1):011502.

31. Alitalo K, Carmeliet P. Molecular mechanisms of lymphangiogenesis in health and disease. Cancer Cell. 2002;1(3):219-27.

32. Schmid-Schonbein GW. Microlymphatics and lymph flow. Physiol Rev. 1990;70(4):987-1028.

33. Jain RK. Transport of molecules in the tumor interstitium: a review. Cancer Res. 1987;47(12):3038-51.

34. Avery M, Nathanson SD, Hetzel FW. Lymph flow from murine footpad tumors before and after sublethal hyperthermia. Radiat Res. 1992;132(1):50-3.

35. Padera TP, Stoll BR, Tooredman JB, Capen D, di Tomaso E, Jain RK. Pathology: cancer cells compress intratumor vessels. Nature. 2004;427(6976):695.

36. Bockhorn M, Jain RK, Munn LL. Active versus passive mechanisms in metastasis: Do cancer cells crawl into vessels, or are they pushed? Lancet Oncol. 2007;8(5):444-8.

37. Willis RA. Pathology of tumours, 3rd edn. London: Butterworth; 1960. p. 167-172.

38. Pressman JJ, Simon MB. Experimental evidence of direct communications between lymph nodes and veins. Surg Gynecol Obstet. 1961;113:537-41.

39. Borodin YI, Tomchek GV. Functional relationships between blood vessels and lymphatic sinuses normally and during experimental disturbances of blood and lymph circulation. Fed Proc Transl Suppl. 1966;25(5):778-80.

40. Bron KM, Baum S, Abrams HL. Oil embolism in lymphangiography. Incidence, manifestations, and mechanisms. Radiology. 1963;80(2):194-202.

41. Hellman S. Karnovsky memorial lecture. Natural history of small breast cancers. J Clin Oncol. 1994;12(10):2229-34.

Publisher's Note Springer Nature remains neutral with regard to jurisdictional claims in published maps and institutional affiliations. 